\title{
Une journée « Science et Médlias » au Palais de la découverte
}

Commission «Culture scientifique » de la Société Française de Physique

Le 9 janvier 2012 s'est tenue, au Palais de la découverte, une journée « Science et Médias, mieux travailler ensemble ». Elle était organisée par la Commission Culture scientifique de la Société Française de Physique (SFP), en partenariat avec I'Association des journalistes scientifiques de la presse d'information (AJSPI).

Le but était de chercher des pistes pour une meilleure collaboration entre ces deux communautés dont l'entente est loin d'être parfaite : les scientifiques se plaignent souvent du manque d'intérêt des médias pour la science, et les journalistes, du fait que les scientifiques sont retranchés dans leur tour d'ivoire.

Il n'était pas prévu de discussion sur l'information scientifique via le web. Nous présentons ci-après un bref compte rendu des trois conférences de la matinée, consacrées à "l'état des lieux ", et des trois tables rondes qui ont suivi.

La journée, dont le programme est donné page 27, a rassemblé 170 personnes (hormis les organisateurs), dont environ 50 chercheurs et enseignants-chercheurs, 50 chargés de communication, une trentaine de journalistes et 24 retraités.

\section{L'AJSPI}

Créée en 1955, I'Association des journalistes scientifiques de la presse d'information (AJSPI) est une association sans but lucratif (loi de 1901), qui a pour objet de rassembler les journalistes professionnels spécialisés dans l'information scientifique et technique.

L'Association entend en particulier entreprendre toute action destinée à faire reconnaître sa place à l'information scientifique dans les médias généralistes, et favoriser la meilleure collaboration possible entre chercheurs et journalistes.

Très active en dépit d'une structure légère et bénévole, elle organise des réunions, des débats entre ses membres, des colloques, des rencontres avec des chercheurs, des voyages... dans un climat confraternel et convivial. L'AJSPI est actuellement forte de 250 adhérents (dont $44 \%$ de pigistes), représentant 75 médias : presse écrite, radio, télévision, presse Internet... C'est la troisième plus grosse association de journalistes scientifiques d'Europe.

Adresse : 102, avenue des Champs-Élysées, 75008 Paris

Site internet: www.ajspi.com

\section{Conférences, président de séance Daniel Bideau}

La première conférence, donnée par Étienne Mercier, rendait compte d'une enquête menée pour La Recherche et Le Monde en juin 2011 sur "Les Français et la science " [1]. La science conserve la confiance du grand public, pour « apporter des solutions aux problèmes rencontrés dans le monde et pour améliorer leur vie ". Cependant, cette confiance dans les scientifiques varie fortement en fonction du domaine, et du risque qui lui est associé. En particulier, sans surprise, le nucléaire est considéré comme un risque d'une extrême gravité et dont la probabilité avérée génère désormais une forte défiance. Les OGM présenteraient un risque non avéré, mais seraient de faible utilité. $86 \%$ des personnes interrogées sont persuadées que l'homme a un impact important sur le changement climatique. Enfin, il y aurait de réelles attentes à l'égard de la communauté scientifique pour expliquer les enjeux de la recherche et les débats qu'elle suscite.

La deuxième conférence, de Robert Nardone, portait surtout sur la radio et la télévision. Pour ce dernier média, une étude de l'Institut national de l'audiovisuel montre que la science a fait l'objet, en une année, de 570 sujets toutes chaines confondues, soit trois fois moins que les sports. Les journaux des deux principales chaînes font surtout état de catastrophes naturelles ou d'erreurs médicales. Dans les interviews, on invite peu les gens qui réfléchissent quand ils parlent (car ils sont jugés trop lents). Et les « experts » sont ceux qui sont les plus « radiogéniques » ou "télégéniques ", c'est-à-dire presque toujours les mêmes. Le conférencier cite alors une émission "mauvaises ondes » sur FR3, où la publicité avant l'émission était tellement orientée que deux experts pressentis ont refusé d'y participer. Dans la même veine, pour lui, la télévision transmet essentiellement de l'émotion et non de la raison.

Le troisième conférencier était Pierre-Henri Gouyon. Pour lui, les scientifiques ont besoin de publier dans des revues reconnues ou des magasines (Nature...) et même dans la presse quotidienne, le financement de leur recherche dépendant essentiellement de leur reconnaissance. Il y a souvent dans les articles de presse confusion entre science et technique (les chercheurs insistant eux-mêmes sur les applications potentielles de leurs travaux), et mise en exergue de controverses plus sociales que scientifiques (exemple : les OGM). Le traitement récent de la controverse sur l'origine du changement climatique par les médias n'a pas vraiment favorisé l'honnêteté scientifique, mais plutôt le bruit. Les grandes chaînes publiques devraient avoir un conseil scientifique. Nombre d'informations scientifiques sont biaisées : voir "les marchands de doute "( $($ ) ou les fondations financées par les industriels.

(^) Marchands de doute : un petit groupe d'« experts indépendants » et de médias naïs et complaisants, qui discréditent la science et les scientifiques dans le but d'éviter toute réglementation de santé publique ou environnementale. Voir le livre de N. Oreskes et E.M. Conway, Les marchands de doute, Éditions Le Pommier (2012). 


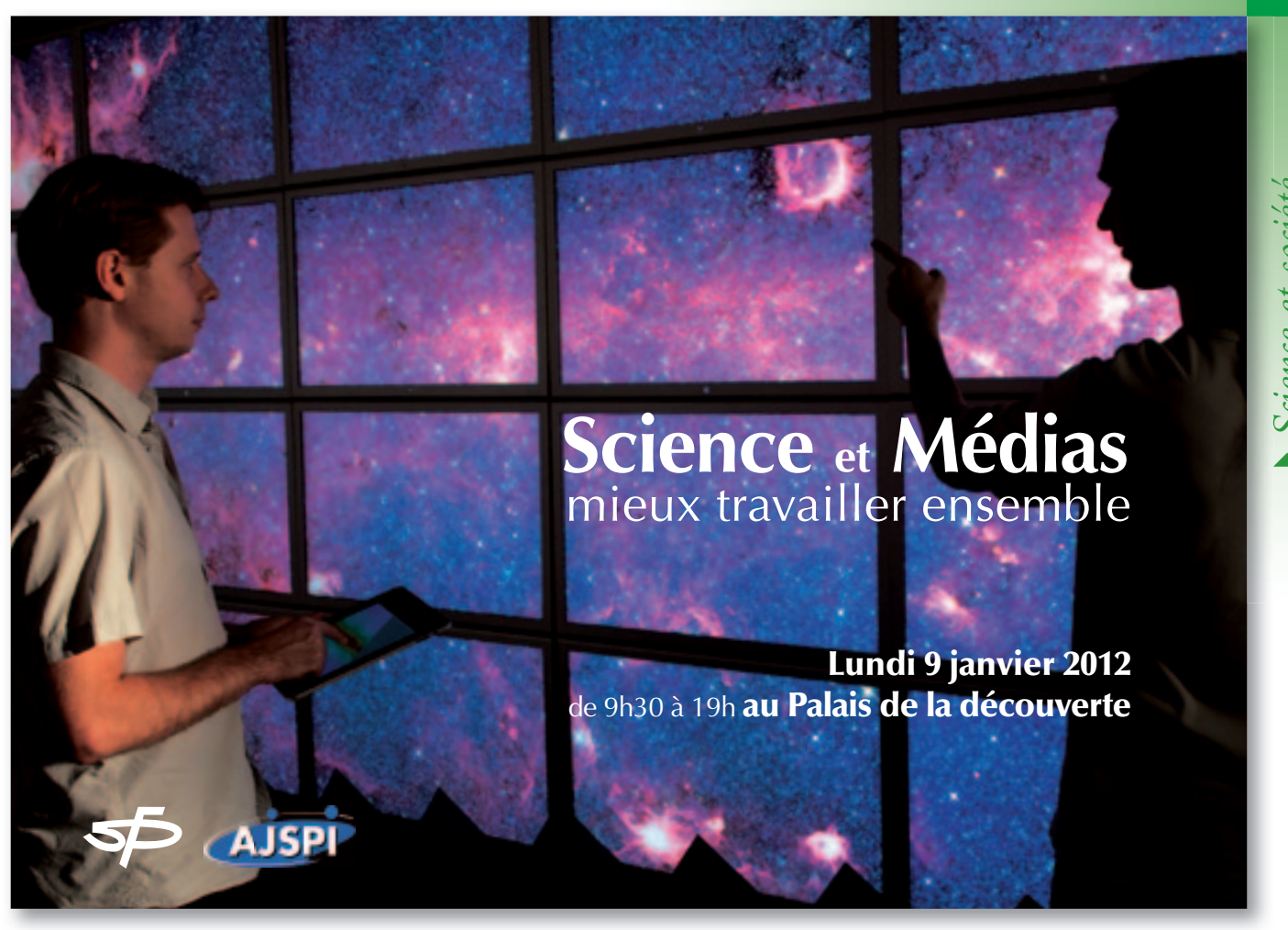

\section{Programme de la journée "Science et Médias, mieux travailler ensemble "}

9h30-10h : $\quad$ Ouverture par Sophie Bécherel, présidente de l'AJSPI, et Martial Ducloy, président de la SFP

$10 \mathrm{~h}-11 \mathrm{~h} 30$ : $\quad$ Conférences

- Les Français et la science, par Étienne Mercier, directeur adjoint du département Opinions de l'IPSOS

- Quelle image de la science les médias véhiculent-ils ?, par Robert Nardone, documentariste de la télévision

- Écologie et évolution face aux créationnistes et aux marchands de doute, par Pierre-Henri Gouyon, spécialiste en science de l'évolution, professeur au Muséum national d'Histoire naturelle

$11 \mathrm{~h} 30-12 \mathrm{~h} 30: \quad$ Table ronde 1

- Entre le marteau et l'enclume, animée par Gérard Torchet (SFP)

Muriel Florin, formation littéraire, journaliste au Progrès de Lyon

Yvon Lechevestrier, ancien journaliste à Ouest-France

Cécile Michaut, docteur en chimie, journaliste scientifique à Sciences et Avenir

14h-15h30: $\quad$ Table ronde 2

- Témoignages, animée par Patrick Hesters (France Télévisions)

Nathalie Blanc, journaliste scientifique à la revue mensuelle Sciences Ouest de l'Espace des Sciences de Rennes

Roland Lehoucq, astrophysicien, CEA et École polytechnique

Jean-François Mathiot, physicien des particules, CNRS et Université de Clermont-Ferrand

Stéphanie Meyer-Thomas, responsable de la communication institutionnelle à l'Université de Strasbourg

Lydie Valade, CNRS et Université Paul Sabatier de Toulouse, présidente de la commission "Chimie et Société » de la Fondation de la Maison de la Chimie

16h $15-17 \mathrm{~h} 45: \quad$ Table ronde 3

- Mieux travailler ensemble, former et informer, animée par Guillaume Trap (Palais de la découverte)

Sophie Bécherel, journaliste généraliste et scientifique à France Inter

Jean-Michel Courty, professeur à l'Université Paris 6, conseiller scientifique à l'Institut de Physique du CNRS

Julien Guillaume, chef du bureau de presse et directeur adjoint de la communication du CNRS

Jean Jouzel, géochimiste au CEA, climatologue, membre du bureau du GIEC (Groupe d'experts intergouvernemental sur l'évolution du climat)

Dominique Leglu, docteur en physique subatomique, directrice de rédaction à Sciences et Avenir Bernard Maitte, cristallographe, historien des sciences, professeur émérite à l'Université de Lille 1

17 h 45 -18h : $\quad$ Conclusion par Daniel Bideau, président de la Commission Culture scientifique de la SFP 


\section{Table ronde « Entre le marteau et l'enclume », animée par Gérard Torchet}

Comment choisit-on un sujet à traiter? Quelles difficultés se présentent et comment s'en sortir ? Trois journalistes témoignent. Un leitmotiv : notre métier, c'est informer, nous ne sommes ni des communicateurs ni des médiateurs.

Muriel Florin. Ne pas avoir de formation scientifique est à la fois un handicap, à cause du temps nécessaire à la compréhension, et un avantage, car on est capable de se mettre à la place du lecteur grand public. Dans un quotidien régional, peu ont une seule thématique en charge (contrairement aux quotidiens nationaux). La plupart des journalistes sont répartis selon des secteurs géographiques.

Mon terrain est vaste : études, famille, enfance... Les sciences sont abordées à travers l'aspect recherche : il s'agit de valoriser les avancées obtenues dans la région et d'informer sur l'actualité. Les dinosaures constituent, pour les lecteurs, un sujet qui disposera de plus de place dans le journal que le boson de Higgs, sujet "moins sexy " (terme utilisé dans les rédactions), pour lequel je me suis battue et n'ai obtenu que 800 signes en bas de page... Une motivation personnelle, c'est la curiosité : "comment ça marche ?". Poser des questions simples. Par exemple, pendant l'été, "pourquoi les orties piquent »... et trouver des scientifiques pour répondre !

Nous sommes aussi soumis à une sorte d'audimat. Les enquêtes révèlent que les lecteurs lisent d'abord les faits divers et le sport, même s'ils disent qu'ils s'intéressent surtout aux questions de société...

Yvon Lechevestrier. Une anecdote. Ouest-France a abordé récemment le sujet des squelettes de Téviec, découverts à Quiberon vers 1920, dans un article accompagné par une vidéo sur son site internet. Ce jour-là, ce fut le sujet le plus lu et le plus vu, parmi les sujets d'actualité. Le grand public peut donc être intéressé par une information scientifique.
Entre le marteau et l'enclume ? J'ai été plutôt bien accueilli et n'ai pas rencontré trop de problèmes... Nous, journalistes, sommes des « conteurs d'histoires " : devant une question scientifique, nous cherchons la personne qui va nous permettre de raconter l'histoire.

Les quotidiens du groupe Ouest-France, ce sont en fait 45 éditions, et environ 40 pages pour une édition : 12 pages communes générales, le reste local ou régional ; on cherche donc d'abord l'information locale, ce qui nécessite de faire un choix entre une info nationale, type « année mondiale de la chimie », et les informations locales qu'attendent les lecteurs.

Cécile Michaut. Dans la presse scientifique (La Recherche, Science et Avenir...), on n'a pas besoin de convaincre le rédacteur en chef de l'intérêt des sujets scientifiques. Mais pour les "piges ", il faut montrer et justifier l'intérêt pour tel sujet. Convaincre pour gagner sa vie...

Un bon sujet a trait à l'actualité : une information (une explosion...) ou ce qui se prépare dans les laboratoires. Certes, on peut alors traiter de sujets ardus (boson de Higgs). Un bon sujet, c'est aussi celui qui touche le lecteur : santé, environnement, métaphysique (l'Univers...). Le scientifique consulté nous aide à remettre un sujet dans un contexte : son intérêt, son avenir... La moitié des chercheurs français sollicités ne répondent pas aux courriels. Les Anglo-Saxons répondent à $95 \%$ et, s'ils ne savent pas, ils proposent des noms de collègues à joindre. Pour les relectures d'articles, les journalistes sont réticents par manque de temps, tandis que les chercheurs souhaitent relire car ils craignent que leurs propos soient déformés.

\section{Table ronde « Témoignages », animée par Patrick Hesters}

Cette deuxième table ronde était consacrée à l'expression de scientifiques et de communicants, sur leur expérience de médiation ou de communication scientifique (deux activités bien distinctes et différentes du métier de journaliste).

Nathalie Blanc travaille à la revue Sciences Ouest [2]. Avec un ancrage régional et très proche des acteurs, elle relate pourtant la difficulté d'entretenir des liens étroits avec la presse quotidienne régionale, même lorsque les manifestations rencontrent de larges succès. À l'Espace des sciences de Rennes (CCSTI rennais), le directeur, Michel Cabaret, témoigne ainsi : "Le succès d'une manifestation ne suffit pas pour diffuser la culture scientifique, pour montrer l'intérêt de la démarche scientifique et le perpétuel questionnement qu'elle nécessite. Pour perdurer, il faut réussir à intéresser à la fois les élus et la presse. "

Roland Lehoucq est extrêmement actif dans le domaine de la diffusion de la connaissance scientifique. L'originalité de sa démarche est l'utilisation de la science-fiction, et des supports de l'imaginaire en général, pour parler de science. Pour lui, une difficulté dans la communication scientifique est de faire passer beaucoup d'informations dans un temps court, alors qu'il y a souvent besoin d'un grand nombre de prérequis. Il faut aussi que les journalistes soient conscients que les scientifiques s'expriment sous le regard de leurs pairs, et qu'après ils vivent avec. Le scientifique a un impératif de vérité, et faire du compréhensible vrai, c'est très difficile.

Jean-Francois Mathiot, physicien théoricien, a de nombreuses activités de popularisation des sciences et de diffusion des connaissances. Il trouve que c'est un exercice difficile, mais très stimulant, de pouvoir adapter son langage à des publics de niveaux très différents. Ce n'est pas seulement une bonne œuvre, le chercheur fait cela pour lui aussi. Intervenir dans les classes, c'est s'adresser aux élèves, mais également trouver des relais via les professeurs. C'est enfin le rôle du chercheur de s'adresser à la société, par des conférences citoyennes locales, sur des sujets de grand intérêt général (par exemple, les OGM).

Stéphanie Meyer-Thomas développe des programmes pour la télévision locale de Strasbourg, visant à améliorer la visibilité des activités de recherche de l'université. Au-delà de l'action de communication se trouvent le rôle de vigie pour alerter les rédactions et la part de conseil pour les chercheurs qui sont en contact avec les journalistes. Pour elle, la communication scientifique est le pont nécessaire entre les journalistes et les scientifiques.

Lydie Valade a une forte expérience de travail avec les médias, acquise à travers les rencontres « Chimie et Terroir » [3] qu'elle a initiées. Ses rapports avec les journalistes ne sont pas forcément difficiles, mais compliqués en raison de l'image négative de la chimie. Elle a mené, par exemple, une série intitulée "le petit chimiste illustré " avec la Dépêche du Midi, qui a très bien marché. Pour autant, certaines actions remarquables et ambitieuses, comme la création d'une affiche géante du tableau périodique des éléments, peinent à attirer les journalistes dans une ville qui reste traumatisée par le souvenir de l'explosion d'AZF. 


\section{Table ronde « Mieux travailler ensemble, former et informer », animée par Guillaume Trap}

Le but de cette table ronde était d'analyser les difficultés de communication entre scientifiques et journalistes, et de voir les moyens d'y remédier. Mais attention : comme l'ont souligné Dominique Leglu et Bernard Maitte, les lignes éditoriales des différents médias ne sont pas les mêmes, et les scientifiques (individus, équipes ou institutions) n'ont pas tous les mêmes intérêts.

\section{Les difficultés}

- Les contraintes de temps sont différentes chez les journalistes et les scientifiques.

Guillaume Trap rappelle un constat de Pierre Desgraupes : «L'information scientifique transmise par les médias résulte d'un compromis entre deux démarches antagonistes par essence : d'un côté celle des scientifiques, tenus par une exigence de rigueur qui les incline naturellement à ne divulguer les progrès de leurs recherches qu'avec une extrême prudence ; de l'autre côté, il y a des journalistes qui, dressés à chasser le scoop en toute occasion, ont peine à se plier à cette exigence de retenue. "

- Souvent, les journalistes ne savent pas comment fonctionne la science. Sophie Bécherel rapporte une anecdote significative. "Le lendemain de l'annonce des neutrinos supraluminiques, on a invité à la rédaction un physicien, qui a insisté sur le fait que lui-même était très étonné de ce résultat, et qu'il faudrait évidemment le reproduire. Cela a soulevé un tollé pour la moitié des journalistes présents, parce qu'ils n'ont pas compris pourquoi un scientifique venait nuancer à ce point les résultats qu'on avait annoncé le matin même comme tout à fait extraordinaires. »

- Journalistes et scientifiques ont des logiques différentes.

Le journaliste ne prêche pas la cause de la science, mais il informe et n'a pas à prendre parti. "À l'AJSPI, nous ne sommes pas là pour dire que la science c'est le progrès, qu'elle est forcément bonne, que c'est notre avenir. Nous sommes les porte-parole des sciences, mais avec tout ce qu'elles comportent comme controverses, dimensions sociétale, politique, économique..., contrairement évidemment aux communicants ou aux médiateurs. "

- Les journalistes donnent-ils trop de place aux "marchands de doute" dans les médias?

Jean Jouzel pense que c'est inéluctable. "Prenons l'exemple du réchauffement climatique. Si vous acceptez que c'est un problème potentiel qui nous emmène forcément vers une société sobre en carbone, cela a tellement d'importance sur notre vie de tous les jours et notre mode de développement, que c'est normal qu'on soit questionnés. On ne peut pas y échapper."

Mais c'est dans le choix des interlocuteurs qu'on peut faire des reproches aux journalistes : pour eux, s'il n'y pas de débat, il n'y a pas d'audience.

\section{Pour mieux travailler ensemble, former les scientifiques, mais aussi les journalistes}

Jean-Michel Courty a vu une réelle évolution depuis 5 ans. "Au début, la plupart des gens étaient assez réticents à faire de la communication. Et puis, avec les modes de financement sur projets, de plus en plus de laboratoires font remonter des textes pour qu'on parle de ce qu'ils ont fait, mais, hélas, ils le font parfois en se prenant pour des publicitaires. Donc, on essaie de recadrer les choses. " Pour cela, la cellule de communication de l'INP du CNRS met au point des formations de deux jours, au Centre de vulgarisation de la connaissance à Orsay; là, on décrit au scientifique ce qu'est un journaliste et comment fonctionnent les différents médias. On lui explique qu'il faut intéresser le public, mais éviter la survente.

Sophie Bécherel rappelle que, de son côté, l'AJSPI organise depuis 9 ans des "Bourses d'échange chercheur-journaliste ", qui consistent à ce qu'un scientifique vienne une semaine dans une rédaction et à ce qu'un journaliste aille passer une semaine dans un laboratoire. L'idée est de partager la vie du chercheur ou du journaliste, pour en percevoir les contraintes. C'est extrêmement formateur et les retours sont très positifs.
Bernard Maitte, ancien professeur à l'École supérieure de journalisme de Lille, évoque les formations de journalistes scientifiques, où les élèves ont des stages à faire dans la presse, dans des laboratoires et dans des structures de communication de grands organismes. Voir comment ces logiques fonctionnent et quelles sont les contraintes réciproques des uns et des autres amène à se parler, se comprendre et se respecter.

Une discussion s'ensuit sur le media training. Dominique Leglu n'aime pas l'idée que cela pourrait amener à du formatage, et est convaincue qu'il faut conserver une certaine spontanéité, des prises de position personnelles. Elle insiste sur l'enrichissement que constitue pour le chercheur l'interaction directe avec un journaliste. Julien Guillaume répond qu'au contraire, le media training va permettre au chercheur d'être plus spontané. L'objectif de ces formations est que tout chercheur, et pas seulement ceux qui ont un talent de "bon client ", puisse répondre " oui » à la demande d'un journaliste. Il est important de faire émerger de "nouvelles têtes ", de nouveaux sujets, auprès des journalistes.

\section{Conclusion}

In fine, cette journée a permis des échanges souvent intéressants entre diverses communautés, l'assistance étant pour partie importante composée de représentants de la communication scientifique.

Un certain nombre de propositions ont été faites lors des discussions. En particulier, une partie du public insistait sur l'importance actuelle de l'information scientifique sur Internet. Cela pourrait être le thème d'une prochaine rencontre.

Il serait vain de prétendre que cette journée a abattu des obstacles menant à une information scientifique satisfaisant toutes les parties prenantes. Mais, comme souvent, on peut espérer que le fait de se rencontrer, d'échanger et de mieux se connaitre permettra de meilleures relations entre les sociétés savantes et les médias. La connaissance par chaque partenaire des contraintes des différents métiers (par exemple leurs temps caractéristiques) est en effet indispensable à ces bonnes relations.

Daniel Bideau (daniel.bideau@univ-rennes1.fr), Charles de Novion, Paul-Éric Pottie et Gérard Torchet

\section{Références}

